\title{
Abordaje vaginoscópica para la realización de histeroscopía oficinal: experiencia, resultados y revisión de la literatura
}

\author{
Rodrigo Carvajal G. ${ }^{1}$, Armando Cortínez $C .^{1}$, Constanza Soto L. ${ }^{a}$, Cristián Miranda V. ${ }^{1}$ \\ Antonio Carvajal M. ${ }^{1}$, Iván Gallegos M. ${ }^{2}$, David Vantman B. ${ }^{1}$ \\ ${ }^{1}$ Unidad de Medicina Reproductiva e Infertilidad, Departamento de Obstetricia y Ginecología. ${ }^{2}$ Departamento de Anato- \\ mía Patológica. Hospital Clínico Universidad de Chile.
}

a Alumna, Escuela de Medicina, Universidad Católica del Maule.

\section{RESUMEN}

Objetivo: Presentar nuestra experiencia y una revisión de la literatura sobre la utilización de la vaginohisteroscopia oficinal. Describir las principales indicaciones, su tolerancia, los procedimientos realizados y las complicaciones resultantes. Método: Estudio descriptivo de todos los procedimientos vaginohisteroscópicos ambulatorios realizados en la Unidad de Medicina Reproductiva e Infertilidad del Departamento de Obstetricia y Ginecología del Hospital Clínico de la Universidad de Chile, entre el $1^{\circ}$ de enero de 2008 y el 31 de enero de 2014. Resultados: Se realizaron un total de 632 vaginohisteroscopias: $63,7 \%$ diagnósticas y $36,2 \%$ quirúrgicas. El tiempo promedio de realización fue de $13 \pm 8$ minutos. La percepción de dolor presentó una mediana de EVA 4 con intercuartiles de 3 y 6 . La principal indicación fue por sospecha de pólipos endometriales (33\%) y engrosamiento endometrial en un 12\%. El hallazgo histeroscópico más frecuente fue cavidad endometrial normal (27\%) y pólipos endometriales $(27 \%)$. Los procedimientos más frecuentes fueron polipectomías (27\%) y biopsias endometriales (20\%). Se registraron 17 complicaciones (2,6\%): 14 reacciones vasovagales $(2,2 \%)$ y 3 lipotimias $(0,4 \%)$. Conclusiones: El abordaje vaginohisteroscópico ha demostrado ser una técnica segura, resolutiva, bien tolerada por las pacientes y de menor costo.

\section{PALABRAS CLAVE: Vaginohisteroscopia, histeroscopia oficinal, histeroscopia ambulatoria, histeroscopia diagnóstica}

\section{SUMMARY}

Objective: To present our experience and a review of the literature of the vaginohysteroscopic technique. To describe the main indications, the tolerance, the procedures performed and their complications. Method: This is a descriptive study of all outpatient procedures performed with the vaginohysteroscopic technique in the Reproductive Medicine and Infertility Unit of the Department of Obstetrics and Gynecology at University Hospital of Chile between 1st January of 2008 and 31st January of 2014. Results: A total of 632 vaginohysteroscopys were performed: $63.7 \%$ diagnostic and $36.2 \%$ surgical. The average procedure time was 13 \pm 8 minutes. Pain showed a median of VAS of 4 . The main indication was suspected endometrial polyps $(33 \%)$ and endometrial thickening (12\%). The most frequent finding was normal endometrial cavity $(27 \%)$ and endometrial polyps (27\%). The most common procedures were polypectomy (27\%) and endometrial biopsies (20\%). We registered 17 complications (2.6\%): 14 vasovagal reactions $(2.2 \%)$ and 3 lipothymias $(0.4 \%)$. Conclusions: vaginohisteroscopy approach has proven to be a safe and very resolutive technique, well tolerated by patients and considerably cost-effective.

KEY WORDS: Vaginohysteroscopy, office hysteroscopy, outpatient hysteroscopy, diagnostic hysteroscopy 


\section{INTRODUCCIÓN}

El desarrollo de la histeroscopia oficinal ha permitido un abordaje mínimamente invasivo de la vagina, cavidad endometrial y la resolución de sus patologías más frecuentes. Es así, como se ha demostrado su gran efectividad y precisión en la evaluación de la patología endometrial: como el sangrado uterino anómalo, aborto recurrente e infertilidad $(1,2)$. Así mismo, el avance de la técnica ha cambiado el antiguo paradigma de considerar a la histeroscopia diagnóstica a la de resolución ambulatoria y a la quirúrgica como única posibilidad de realizar en pabellón con anestesia. De esta forma, se ha dado lugar al nuevo concepto de histeroscopia oficinal y su revolucionaria estrategia de "diagnosticar y tratar" en un mismo procedimiento y que puede, por lo tanto, ser quirúrgica y ambulatoria al mismo tiempo (3). A través de la histeroscopia oficinal es posible evaluar la cavidad endometrial, visualizar los ostium tubarios y, específicamente mediante el abordaje vaginohisteroscópico, podemos explorar la mucosa vaginal, el exocérvix y estudiar íntegramente en todo su recorrido el canal endocervical (4). De igual modo, se pueden resolver las patologías más frecuentes como pólipos endometriales, pequeños miomas, sinequias uterinas y tabiques uterinos $(5,6)$.

Sin embargo, a pesar de los importantes avances tecnológicos y técnicos en el instrumental histeroscópico con el desarrollo de equipos de cada vez menor diámetro, el empleo de medios de distensión hidrosalinos y el uso de electrodos bipolares, la presencia de dolor, estenosis cervical y la dificultad para la óptima visualización siguen siendo un impedimento para que los ginecólogos opten primariamente por la histeroscopia oficinal como herramienta diagnóstica y terapéutica de las patologías de la cavidad endometrial (7).

Para evitar estas dificultades que producen un desmedro de la popularidad del abordaje oficinal, es imprescindible seleccionar correctamente a la paciente, conocer las indicaciones más adecuadas, familiarizarse con las limitaciones de la técnica y, sobretodo, adquirir las habilidades y destrezas para poder aplicar adecuadamente la técnica vaginohisteroscópica $(3,6)$.

Clásicamente, el abordaje convencional incluye espéculo vaginal y pinzamiento del cérvix para rectificar el útero y mejorar la visualización del orificio cervical externo lo que produce dolor e incomodidad en la paciente. Además, frecuentemente se realiza dilatación cervical, por lo que es necesaria anestesia paracervical o sedación; incluso, se suele aseptizar la vagina y el cuello uterino para asemejarlo a una histeroscopia en quirófano lo que dista de ser mínimamente invasivo (8). La introducción del abordaje vaginohisteroscópico, "no touch technique", ha transformado la técnica en un proce- dimiento menos invasivo y mucho más accesible, ya que evita el uso de espéculo y pinza Pozzi al introducir el histeroscopio bajo visión directa respetando la anatomía normal del canal endocervical disminuyendo así el dolor y mejorando la tolerancia $(9,10)$.

El objetivo de este trabajo es presentar la experiencia en la implementación de la vaginohisteroscopía oficinal y describir las principales indicaciones, la tolerancia al dolor, los procedimientos realizados y las complicaciones que se presentaron a lo largo de 6 años desde su utilización.

\section{PACIENTES Y MÉTODOS}

Estudio descriptivo de todos los procedimientos vaginohisteroscópicos ambulatorios realizados en la unidad de Medicina Reproductiva e Infertilidad del Departamento de Obstetricia y Ginecología del Hospital Clínico de la Universidad de Chile entre el $1^{\circ}$ de Enero de 2008 y 31 Enero de 2014. Todos los procedimientos fueron realizados por el mismo equipo médico y fueron incluidos en una base de datos Filemaker®, especialmente diseñada para el registro prospectivo de los antecedentes clínicos de las pacientes y los resultados obtenidos en sus respectivas histeroscopias. Se consideraron antecedentes como paridad, edad, complicaciones, la dificultad en la realización del procedimiento graduada en una escala de 0-4, la duración del procedimiento histeroscópico, el diagnóstico pre y post histeroscopia, el tipo de procedimiento realizado, el grado de dolor evaluado según escala visual análoga (EVA) entre 1 y 10, y los resultados de las biopsias en los casos correspondientes.

En todos los procedimientos se utilizó un equipo histeroscopia oficinal tipo Bettocchi (Karl-Storz endoskope $($ ) , consistente en una camisa de $5,4 \mathrm{~mm}$ con un canal de trabajo de $5 \mathrm{Fr}$, una óptica de 2,9 $\mathrm{mm}$ de $30^{\circ}$. Como elementos de trabajo se utilizaron únicamente pinzas tipo grasping y tijeras punta redonda ambas de 5Fr. En todos los procedimientos se utilizó como medio de distención solución fisiológica. Para un mejor control de la irrigicación y de la presión de distención se utilizó un equipo automatizado (Endomat, Karl-Storz endoskope $®$ ). La presión de trabajo se fijó a $75 \mathrm{mmHg}$ con un máximo de $100 \mathrm{mmHg}$, el flujo a $200 \mathrm{ml} / \mathrm{min}$ y la succión a 0,25 bar.

Todos los procedimientos histeroscópicos se realizaron ambulatoriamente con la técnica de vaginohisteroscopia. Esta técnica no requiere la utilización de espéculo vaginal ni pinzamiento cervical, sin requerimientos de anestesia, sedación, pabellón ni dilatación cervical. Una vez, la paciente en posición ginecológica, se apoya en la horquilla vulvar la punta del histeroscopio y se distiende la vagina con el medio de distensión realizando primero una vaginoscopia para luego 
avanzar al cérvix e identificar el orificio cervical externo (OCE). Identificado el OCE se accede al canal endocervical y con suaves movimientos de rotación, siempre visualizando el frente de avance para producir menos dolor se logra la entrada a la cavidad endometrial.

El estudio fue aprobado por el Comité de Ética del Hospital Clínico de la Universidad de Chile y para su realización se contó con el apoyo y participación del Servicio de Anatomía Patológica del mismo hospital, que proporcionó una base de datos propia de la cual se obtuvo el correlato anátomopatológico.

Para el análisis estadístico de los datos se usó el programa Graph Pad 5.0®. Las variables continuas se expresaron como media \pm desviación estándar, las discontinuas como mediana \pm intercuartiles y las cualitativas como frecuencia expresada en porcentajes. Como herramientas estadísticas se utilizó Mann-Whitney y test de Fisher. Se determinó como significativo un valor $p<0,05$.

\section{RESULTADOS}

Se realizaron un total de 632 vaginohisteroscopias en el período analizado. La edad media de las pacientes fue de $41 \pm 11$ años (rango: 17-89 años). Un $36 \%(n=228)$ de las pacientes era nulípara y un $63,9 \%(n=404)$ multíparas. Del total de histeroscopias, 403 (63,7\%) fueron diagnósticas y $229(36,2 \%)$ quirúrgicas. El tiempo promedio de realización del procedimiento fue de $13 \pm 8$ minutos (rango: 1-50 minutos). La percepción de dolor en las pacientes se estimó por EVA y presentó una mediana de 4 con intercuartiles de 3 y 6 . El grado de dificultad presentó una mediana de 1 con intercuartiles de 1 y 2.

Las principales indicaciones de la vaginohisteroscopia fueron por sospecha de pólipos endometriales en la ecografía transvaginal en un 33\% $(n=210)$ y engrosamiento endometrial ecográfico en un $12 \%(n=77)$ (Tabla I). Las otras indicaciones médicas fueron menos frecuentes y se presentan en la Tabla I. Los hallazgos histeroscópicos más frecuentes fueron cavidad endometrial normal $27 \%$ $(n=171)$, pólipos endometriales $27 \%(n=173)$ y miomas submucosos $10 \%(n=65)$, otros hallazgos se presentan en la Tabla II.

Se realizaron 229 vaginohisteroscopias quirúrgicas, $36,3 \%$ del total (Tabla III). Los principales procedimientos realizados fueron polipectomías $27 \%(n=62)$, biopsias endometriales $20 \%(n=48)$, extracción de dispositivos intrauterinos 19\% $(n=44)$, dilataciones cervicales $11 \%(n=27)$ y otros procedimientos que se presentan en menor porcentaje.

Como era de esperar la media de tiempo operatorio fue mayor para los procedimientos quirúrgicos; siendo $12,6 \pm 6$ min para las diagnósticas y 15,4 $\pm 8 \mathrm{~min}$ para las operatorias,
Tabla I

INDICACIONES DE LA HISTEROSCOPIA

\begin{tabular}{lc}
\hline Diagnóstico & Pacientes (\%) \\
\hline Pólipos endometriales & $210(33)$ \\
Engrosamiento endometrial & $77(12)$ \\
Infertilidad & $67(10)$ \\
Miomas & $59(9,3)$ \\
Sangrado uterino anómalo & $56(8,8)$ \\
Extracción DIU & $44(6,9)$ \\
Aborto recurrentes & $22(3,3)$ \\
Histerosalpingografía alterada & $21(3,4)$ \\
Tabique uterino & $19(3)$ \\
Essure & $15(2,3)$ \\
Sinequias uterinas & $11(1,7)$ \\
Control postoperatorio & $11(1,7)$ \\
Otras & $20(3,1)$ \\
\hline Total & $632(100 \%)$ \\
\hline
\end{tabular}

Tabla II HALLAZGOS HISTEROSCÓPICOS

\begin{tabular}{lc}
\hline Tipo de patología intrauterina & $\mathrm{n}(\%)$ \\
\hline Normal & $171(27)$ \\
Pólipos & $173(27)$ \\
Miomas & $65(10)$ \\
Engrosamiento endometrial & $51(8)$ \\
Dispositivo intrauterino & $44(6,9)$ \\
Malformación uterina & $43(6,8)$ \\
Otras & $29(4,5)$ \\
Adherencias & $20(3,1)$ \\
Estenosis cervical & $20(3,1)$ \\
Atrofia endometrial & $16(2,5)$ \\
\hline
\end{tabular}

Tabla III PROCEDIMIENTOS HISTEROSCÓPICOS

\begin{tabular}{lc}
\hline Tipo & $\mathrm{n}(\%)$ \\
\hline Diagnósticas & $403(63,7)$ \\
Quirúrgicas: & $229(36,3)$ \\
- Polipectomía & $62(27)$ \\
- Biopsia endometrio & $48(20)$ \\
- Extracción DIU & $44(19)$ \\
- Dilatación cervical & $27(11)$ \\
- Septoplastía & $19(8)$ \\
- Essure & $15(6)$ \\
- Adhesiolisis & $11(4)$ \\
- Otras & $3(1)$ \\
\hline
\end{tabular}


diferencia estadísticamente significativa $(p<0,05)$. Sin embargo, al comparar la percepción del dolor la mediana en los procedimientos quirúrgicos fue EVA 4; mientras que la mediana de EVA en los procedimientos diagnósticos fue EVA 5, diferencia estadísticamente significativa $(p<0,05)$. Esta diferencia en la menor percepción del dolor a favor de los procedimientos quirúrgicos, probablemente se deba a la mayor proporción de multiparidad en este grupo de pacientes. En efecto, una significativa mayor proporción de nulíparas (63\%) se realizaron histeroscopias diagnósticas, lo que explicaría la mayor percepción de dolor, si lo comparamos con el grupo de histeroscopias quirúrgicas $(53 \%$ de nulíparas) $(p<0,05)$.

Se evaluó la percepción del dolor por paridad. La mediana para nulíparas fue EVA 5 y para multíparas EVA 4; sin embargo la menor percepción del dolor por parte de pacientes multíparas no presentó significancia estadística $(p=0,1)$. En este mismo sentido, se contrastaron los efectos de paridad sobre la percepción del dolor y el tiempo operatorio. La media de tiempo operatorio fue interesantemente mayor para el grupo de multíparas (14,3 $47 \mathrm{~min})$ en comparación con las nulíparas (12,9 $77 \mathrm{~min})(\mathrm{p}$ $<0,05)$. Probablemente se explique por una mayor proporción de procedimientos quirúrgicos en las pacientes multíparas (37\%) en comparación con las nulíparas (27\%) ( $p<0,05)$.

Se revisaron los informes de las biopsias de las polipectomías y se encontró que el $21 \%(n=13)$ presentaba hiperplasia sin atipias y el 3,2\% hiperplasia con atipias $(n=2)$. De manera similar, de los engrosamientos endometriales el $20 \%(n=6)$ se informó con hiperplasia $\sin$ atipias y el $6,8 \%(n=2)$ con atipias.

Se registraron un total de 17 complicaciones $(2,6 \%)$ (Tabla IV). Las principales complicaciones fueron 14 reacciones vasovagales $(2,2 \%)$ y 3 lipotimias $(0,4 \%)$. Al analizar si había mayor proporción de complicaciones al agruparlas por paridad (nulípara v/s multípara) o por tipo de vaginohisteroscopía (diagnóstica o quirúrgica) no se encontraron diferencias significativas.

\section{DISCUSIÓN}

La vaginohisteroscopia se ha transformado en el estándar para la evaluación de la cavidad

\section{Tabla IV \\ COMPLICACIONES DURANTE LA HISTEROSCOPÍA}

\begin{tabular}{lc}
\hline Tipo & $\mathrm{n}(\%)$ \\
\hline Reacción vasovagal & $14(2,2)$ \\
Lipotimias & $3(0,4)$ \\
Total & $17(2,6)$ \\
\hline
\end{tabular}

endometrial. Los vertiginosos avances han reducido los calibres de los histeroscopios y la reciente introducción de la energía bipolar han permitido el manejo ambulatorio de la mayoría de las lesiones intracavitarias, sin la necesidad de pabellón, hospitalización, dilatación cervical y anestesia, reduciendo las complicaciones y los costos (3).

Destaca de las características clínicas de las pacientes que son un grupo con amplio rango de edades, con rango entre 17 y 89 años. Además, no se encuentran restringidas solamente a pacientes multíparas y de hecho, un $36 \%$ de las pacientes era nulípara, incluso dentro de nuestra casuística tenemos pacientes sin actividad sexual, por lo que ni la edad ni la paridad son excluyentes como indicación para este tipo de técnica. Por otro lado, es importante destacar que un gran porcentaje de las vaginohisteroscopias fue de tipo diagnóstica $(63,7 \%)$ lo que pudiese explicar la buena tolerancia referida por las pacientes. Este alto porcentaje, puede estar relacionado en parte a que la mayoría de las pacientes fueron referidas por la sospecha no confirmada de patología endometrial sugerida por los hallazgos de una ecografía transvaginal (pólipos endometriales, engrosamiento endometrial o miomas submucosos) o por antecedentes clínicos de infertilidad o aborto a repetición (Tabla I). Por esta razón, y en concordancia con los hallazgos descritos por otros grupos, un $27 \%$ de nuestras pacientes presentaba como hallazgo histeroscópico una cavidad endometrial normal (5).

Del mismo modo, como ha sido descrito por otros autores, los hallazgos histeroscópicos patológicos más frecuentes fueron los pólipos endometriales $(27 \%)$, miomas submucosos (10\%) y engrosamiento endometrial (8\%) (Tabla II) (11). Dentro de las indicaciones de la vaginohisteroscopía es importante recalcar los casos de inserción de ESSUREs, tanto para efectos de anticoncepción definitiva como para el tratamiento de hidrosalpinx en pacientes con deseo de fertilidad y elevados factores de riesgo operatorios y anestésicos (Tabla I) $(12,13)$. Otra indicación cada vez más emergente en la esfera reproductiva, además de la utilidad en el estudio de la pareja infértil, es en parejas asintomáticas que serán sometidas a tratamientos de reproducción asistida. Se ha visto en una reciente revisión sistemática y metaanálisis que aunque no se encuentre patología endometrial, la sola realización de una histeroscopia previa aumenta las tasas de embarazo (NNT=10; IC95\% 7-14) y de nacidos vivo (NNT=11; IC95\% 7-16) lo que apoya la importancia de implementar esta técnica en unidades de reproducción asistida (14).

Por otro lado, destaca el corto tiempo operatorio, a pesar de la presencia de médicos ginecólogos en formación en la mayoría de los procedimientos, con una media de $13 \pm 8$ min para el total de histeroscopias, 12,6 \pm 6 min para las diagnósticas 
y $15,4 \pm 8$ min para las quirúrgicas. Si bien estas diferencias son clínicamente intrascendentes para la práctica diaria, estadísticamente fueron significativas $(p<0,05)$. A pesar de lo anterior, si consideramos la escasa preparación pre-operatoria y post-operatoria, la no necesidad de vía venosa ni anestesia, el uso como recurso de la sala de procedimientos se vuelve muy eficiente y provechoso si lo comparamos con una histeroscopia realizada en pabellón $(15,16)$.

Un $36,3 \%$ de las vaginohisteroscopias oficinales fueron quirúrgicas (Tabla III). Dentro de los principales procedimientos realizados destaca la polipectomía $(27 \%)$, las biopsias endometriales (20\%) y la extracción de DIUs extraviados (19\%). Es interesante destacar que las vaginohisteroscopias quirúrgicas presentaron una mejor tolerancia al dolor $(E V A=4)$ que las histeroscopias diagnósticas $(E V A=5)(p<0,05)$ a pesar del mayor tiempo operatorio y de la realización de un procedimiento operatorio en sí mismo. Creemos que esta diferencia se explica por un mayor porcentaje de las pacientes en el grupo de las histeroscopias diagnósticas era nulípara en comparación con las quirúrgicas $(63 \%$ vs. $53 \%$ ), diferencia que fue estadísticamente significativa. Sin embargo, comparamos la mediana de dolor entre las multíparas y nulíparas, si bien hay una tendencia a percibir menos dolor en el grupo de las multíparas (EVA de 4 vs. 5) no demostramos una diferencia estadísticamente significativa. De esta forma, podemos decir que las histeroscopias quirúrgicas fueron mejor toleradas que las diagnósticas porque hay un mayor porcentaje de pacientes multíparas, y que por el hecho de tener indicación quirúrgica no se traduce en una mayor tasa de fracaso por mala tolerancia, si las comparamos con las exclusivamente diagnósticas.

Mazzon y cols (17), en un estudio prospectivo randomizado evaluó mediante un análisis multivariado los factores que influyen sobre la percepción de dolor encontrando resultados similares; en este estudio se describe que la presencia de sinequia cervical, duración del procedimiento, nuliparidad y uso de medio de distensión salina se asociaban independientemente a un EVA >3. Sin embargo, Craciunas y cols (18), en relación al uso de medio de distensión demuestra en una revisión sistemática y metaanálisis de 10 RCTs que el uso de solución salina produce significativamente menos dolor que el CO2 para histeroscopias diagnósticas. De igual forma, la presión de trabajo que fijamos al medio de distensión afecta la visibilidad y produce dolor. En un estudio randomizado doble ciego, donde se evaluaba el efecto de diferentes presiones de trabajo sobre la visibilidad y dolor, se encontró que por debajo de $40 \mathrm{mmHg}$ se obtiene mala visibilidad y entre 70 y $100 \mathrm{mmHg}$ se obtiene la mejor visibilidad, pero sin diferencias en la percepción del dolor (19).

Según estos resultados y los publicados por otros, la vaginohisteroscopia es la técnica de elección para acceder a la cavidad endometrial por su eficiencia y menor dolor $(9,10)$. Cooper y cols (10), en una revisión sistemática demostró que las pacientes presentaban significativamente menos dolor y mejor tolerancia con igual tasa de accesibilidad a la cavidad endometrial, al comparar el abordaje vaginoscópico vs. histeroscópico tradicional.

El uso de premedicación, como antiinflamatorios no esteroidales (AINEs), es frecuentemente usado antes de los procedimientos histeroscópicos. En una revisión Cochrane, Ahmad y cols (20), sin distinguir entre abordaje vaginoscópico o tradicional, concluye que los anestésicos locales si disminuyen el dolor intraprocedimiento y durante los primeros 30 minutos, aunque no disminuyen el tiempo operatorio ni los eventos adversos. En esta misma revisión, se concluye que los AINEs y los analgésicos opioides no disminuyen significativamente el dolor comparado con placebo; sin embargo interesantemente si disminuyen la suspensión del procedimiento cuando tenía como causal el dolor y no por estenosis cervical (20).

Así mismo, como premedicación hay estudios contrapuestos con respecto al uso de misoprostol previo a la realización de una histeroscopia oficinal. Se ha visto que pudiera disminuir el dolor evaluado por EVA, pero que no aumenta la accesibilidad a la cavidad endometrial ni mejora la satisfacción de la usuaria, produciendo además dolor cólico preoperatorio, fiebre y sangrando $(21,22)$. Sin embargo, otros autores han encontrado en pacientes que consultan por infertilidad que disminuye el tiempo operatorio además del dolor evaluado por EVA $(23,24)$. De esta manera, el uso de misoprostol para histeroscopia oficinal no es una práctica habitual en nuestro grupo por lo anteriormente discutido, pero además, porque tiene el inconveniente práctico que debe administrarse varias horas antes del procedimiento e idealmente vía vaginal, lo que es una desventaja para un procedimiento que realizamos ambulatoriamente en pocos minutos (25). Sin embargo, reconocemos que puede ser beneficioso la utilización de prostaglandinas vía vaginal u oral y estrógenos locales para facilitar el acceso a la cavidad endometrial en los casos de cuellos uterinos muy estenóticos y principalmente en postmenopáusicas (26).

Otra recomendación de buena práctica clínica es citar a las pacientes en fase proliferativa precoz, sobre todo si se realizarán procedimientos quirúrgicos, ya que el endometrio tiende a edematizarse dificultando la visualización de la cavidad endometrial. En este sentido, si queremos realizar una histeroscopia diagnóstica no es recomendable el uso de anticonceptivos hormonales, progestinas u análogos de $\mathrm{GnRH}$, ya que si bien induciría un adelgazamiento endometrial, también se alterarían la características y la diferenciación propia de cada 
fase endometrial dificultando el diagnóstico. Sin embargo, si queremos programar una cirugía histeroscópica ambulatoria, si es recomendable hacer una preparación endometrial previa con progestinas solas o anticonceptivos orales combinados que contengan dienogest (27).

La revisión de los informes de anatomía patológica de los procedimientos realizados destaca que el $21 \%$ de los pólipos y el $20 \%$ de los engrosamientos endometriales presentaban hiperplasia, de las cuales un $3,2 \%$ y $6,8 \%$ respectivamente presentaron atipias. Rahimi y cols (28), evaluaron la asociación de pólipos y atipias endometriales y encontró que un $18 \%$ de todas las pacientes presentaban hiperplasia endometrial y un 7,3\% atipias celulares. En el mismo trabajo, al analizar sólo las pacientes postmenopáusicas un $21,6 \%$ presentaba hiperplasia, $12 \%$ atipias y un $1,2 \%$ adenocarcinoma asociado, subrayando el riesgo de malignización en este grupo de pacientes (28). Si bien no reportamos casos de adenocarcinoma en nuestra casuística, este riesgo existe y es variable en la literatura entre 3,5-3,8\%, dependiendo del estado menopáusico y metrorragia principalmente $(29,30)$.

Las complicaciones de la vaginohisteroscopia son raras y menores, en nuestra experiencia se presentaron sólo en el 2,6\% del total de pacientes (Tabla IV). Principalmente, se debieron a reacciones vasovagales secundarias a dolor que no se asociaron significativamente a la paridad o al tipo de procedimiento realizado, resultados similares a los presentados por otros autores $(5,31)$. En un RCT multicéntrico, Campos y cols (31), demuestran que con histeroscopios de 3,5 mm (mini-histeroscopios) al compararlos con histeroscopios tradicionales (5 $\mathrm{mm}$ de diámetro) se produce menos dolor, mayor accesibilidad a la cavidad endometrial y menor tasa de complicaciones (0-3\% de complicaciones), sin importar la experiencia del cirujano ni paridad de la paciente, lo que apoya la reproducibilidad de la técnica y la importancia de contar con instrumental adecuado. Sin embargo, en nuestra experiencia hemos notado la importancia del entrenamiento previo en modelos y simuladores para disminuir la curva de aprendizaje, adquirir mayor seguridad y destrezas al momento de enfrentar al paciente real, lo que optimiza los resultados y genera aprecio por la técnica. Es así que en base a nuestros resultados podemos decir que la vaginohisteroscopia es un procedimiento muy seguro, con baja tasa de complicaciones y que no debe contraindicarse su empleo ambulatorio aún cuando realicemos procedimientos quirúrgicos.

\section{CONCLUSIONES}

El abordaje vaginohisteroscópico ha demostrado ser una técnica segura, resolutiva y bien tolerada por las pacientes. Además, con ella es posible resolver las patologías endouterinas más frecuentes ambulatoriamente, sin pabellón ni anestesia, con una baja tasa de complicaciones y considerables menores costos.

\section{REFERENCIAS}

1. Angioni S, Loddo A, Milano F, Piras B, Minerba L, Melis GB. Detection of benign intracavitary lesions in postmenopausal women with abnormal uterine bleeding: a prospective comparative study on outpatient hysteroscopy and blind biopsy. J Minim Invasive Gynecol 2008;15:87-91.

2. Revel A, Shushan A. Investigation of the infertile couple: hysteroscopy with endometrial biopsy is the gold standard investigation for abnormal uterine bleeding. Hum Reprod 2002;17:1947-9

3. Bettocchi S, Ceci O, Di Venere R, Pansini MV, Pellegrino A, Marello $F$, et al. Advanced operative office hysteroscopy without anaesthesia: analysis of $501 \mathrm{ca}-$ ses treated with a $5 \mathrm{Fr}$ bipolar electrode. Hum Reprod 2002;17:2435 -8.

4. Pérez-Medina T, Bajo JM, Martinez-Cortes L, Castellanos P, Perez de Avila I. Six thousand office diagnostic-operative hysteroscopies. Int J Gynecol Obstet 2000;71:33-8.

5. Van Kerkvoorde TC, Veersema S, Timmermans A. Long-term complications of office hysteroscopy: analysis of 1028 cases. J Minim Invasive Gynecol 2012;19:494-7.

6. Di Spiezio Sardo A, Taylor A, Tsirkas P, Mastrogamvrakis G, Sharma M, Magos A. Hysteroscopy: a technique for all? Analysis of 5,000 outpatient hysteroscopies. Fertil Steril 2007;89:438-43.

7. Readman E, Maher PJ. Pain relief and outpatient hysteroscopy: a literature review. J Am Assoc Gynecol Laparosc 2004;11:315-9.

8. Bettocchi S, Nappi L, Ceci O, Selaggi L. Office hysteroscopy. Obstet Gynecol Clin North Am 2004;31:64154.

9. Cicinelli E, Parisi C, Galantino P, Pinto V, Barba B, Schonauer S. Reliability, feasibility, and safety of minihysteroscopy with a vaginoscopic approach: experience with 6000 cases. Fertil Steril 2003;80:199-202.

10. Cooper N, Smith P, Khan K, Clark T. Vaginoscopic approach to outpatient hysteroscopy: a systematic review of the effect on pain. BJOG 2010;117:532-9.

11. Carabias $P$, Celades-Filella M, Zapardiel I, Alsina-Maqueda A, Genover-Llimona E. Experience and results of office hysteroscopy at a primary hospital. J Obstet Gynaecol 2014;34:54-6.

12. Hurskainen R, Hovi SL, Gissler M, Grahn R, Kukkonen-Harjula K, Nord-Saari M, Mäkelä M. Hysteroscopic tubal sterilization: a systematic review of the Essure system. Fertil Steril 2010;94:16-9.

13. Arora P, Arora RS, Cahill D. Essure $(\circledR)$ for management of hydrosalpinx prior to in vitro fertilisationa systematic review and pooled analysis. BJOG 2014;121:527-36.

14. Pundir J, Pundir V, Omanwa K, Khalaf Y, El-Toukhy T. Hysteroscopy prior to the first IVF cycle: a systematic review and meta-analysis. Reprod Biomed Online 2014;28:151-61.

15. Hidlebaugh D. A comparison of clinical outcomes and 
cost of office versus hospital hysteroscopy. J Am Assoc Gynecol Laparosc 1996;4:39-45.

16. Moawad NS, Santamaria E, Johnson M, Shuster J. Cost-effectiveness of office hysteroscopy for abnormal uterine bleeding. JSLS 2014;18(3).pii: e2014.00393.

17. Mazzon I, Favilli A, Grasso M, Horvath S, Bini V, Di Renzo GC, Gerli S. Pain in diagnostic hysteroscopy: a multivariate analysis after a randomized, controlled trial. Fertil Steril 2014;102:1398-403.

18. Craciunas L, Sajid MS, Howell R. Carbon dioxide versus normal saline as distension medium for diagnostic hysteroscopy: a systematic review and metaanalysis of randomized controlled trials. Fertil Steril 2013;100:1709-14.

19. Shahid A, Pathak M, Gulumser C, Parker S, Palmer E, Saridogan E. Optimum uterine filling pressure for outpatient diagnostic hysteroscopy: a double-blind, randomized controlled trial. Reprod Biomed Online 2014;28:86-91.

20. Ahmad G, O'Flynn H, Attarbashi S, Duffy JM, Watson A. Pain relief for outpatient hysteroscopy. Cochrane Database Syst Rev 2010;(11):CD007710.

21. Singh N, Ghosh B, Naha M, Mittal S. Vaginal misoprostol for cervical priming prior to diagnostic hysteroscopy--efficacy, safety and patient satisfaction: a randomized controlled trial. Arch Gynecol Obstet 2009;279:37-40.

22. Hassa H, Aydin Y, Oge T, Cicek K. Effectiveness of vaginal misoprostol and rectal nonsteroidal anti-inflammatory drug in vaginoscopic diagnostic outpatient hysteroscopy in primarily infertile women: doubleblind, randomized, controlled trial. J Minim Invasive Gynecol 2013;20:880-5.

23. Sordia-Hernández LH, Rosales-Tristan E, VazquezMendez J, Merino M, Iglesias JL, Garza-Leal JG, Morales $A$. Effectiveness of misoprostol for office hysteroscopy without anesthesia in infertile patients. Fertil Steril 2011;95:759-61.

24. El-Mazny A, Abou-Salem N. A double-blind randomized controlled trial of vaginal misoprostol for cervical priming before outpatient hysteroscopy. Fertil Steril 2011;96:962-5.

25. Polyzos NP, Zavos A, Valachis A, Dragamestianos C, Blockeel C, Stoop D, Papanikolaou EG, Tournaye $H$, Devroey P, Messinis IE. Misoprostol prior to hysteroscopy in premenopausal and post-menopausal women. A systematic review and meta-analysis. Hum Reprod Update 2012;18:393-404.

26. Gkrozou F, Koliopoulos G, Vrekoussis T, Valasoulis G, Lavasidis L, Navrozoglou I, Paschopoulos M. A systematic review and meta-analysis of randomized studies comparing misoprostol versus placebo for cervical ripening prior to hysteroscopy. Eur J Obstet Gynecol Reprod Biol 2011;158:17-23.

27. Bifulco G, Di Spiezio Sardo A, De Rosa N, Greco E, Spinelli M, Di Carlo C, Tommaselli GA, Nappi C. The use of an oral contraceptive containing estradiol valerate and dienogest before office operative hysteroscopy: a feasibility study. Gynecol Endocrinol 2012;28:949-55.

28. Rahimi S, Marani C, Renzi C, Natale ME, Giovannini $\mathrm{P}$, Zeloni R. Endometrial polyps and the risk of atypical hyperplasia on biopsies of unremarkable endometrium: a study on 694 patients with benign endometrial polyps. Int J Gynecol Pathol 2009;28:522-8.

29. Wethington SL, Herzog TJ, Burke WM, Sun X, Lerner JP, Lewin SN, Wright JD. Risk and predictors of malignancy in women with endometrial polyps. Ann Surg Oncol 2011;18:3819-23.

30. Baiocchi G, Manci N, Pazzaglia M, Giannone L, Burnelli L, Giannone E, Fratini D, Di Renzo GC. Malignancy in endometrial polyps: a 12-year experience. Am J Obstet Gynecol 2009;201(5):462.e1-4.

31. Campo R, Molinas CR, Rombauts L, Mestdagh G, Lauwers M, Braekmans P, Brosens I, Van Belle $Y$, Gordts S. Prospective multicentre randomized controlled trial to evaluate factors influencing the success rate of office diagnostic hysteroscopy. Hum Reprod 2005;20:258-63. 